\title{
Analyses of Potential Driver and Passenger Bacteria in Human Colorectal Cancer
}

This article was published in the following Dove Press journal:

Cancer Management and Research

\author{
Yijia Wang ${ }^{1, *}$ \\ Chunze Zhang ${ }^{2, *}$ \\ Shaobin $\mathrm{Hou}^{3}$ \\ Xiaojing Wu' \\ Jun Liu ${ }^{4}$ \\ Xuehua Wan (iD $^{5}$
}

'Laboratory of Oncologic Molecular Medicine, Tianjin Union Medical Center, Nankai University, Tianjin, People's Republic of China; ${ }^{2}$ Department of Colorectal Surgery, Tianjin Union Medical Center, Nankai University, Tianjin, People's Republic of China; ${ }^{3}$ Advanced Studies in Genomics, Proteomics, and Bioinformatics, University of Hawaii at Manoa, Honolulu, HI, USA; ${ }^{4}$ Department of Radiology, Tianjin Union Medical Center, Nankai University, Tianjin, People's Republic of China; ${ }^{5}$ TEDA Institute of Biological Sciences and Biotechnology, Nankai University, TEDA, Tianjin, People's Republic of China

*These authors contributed equally to this work
Introduction: Besides genetic and epigenetic alterations that lead to carcinogenesis and development of colorectal cancer (CRC), intestinal microbiomes are recently recognized to play a critical role in CRC progression. The abundant species associated with human CRC have been proposed for their roles in promoting tumorigenesis. However, a recent "driverpassenger" model suggests that these CRC-associated species with high relative abundances may be passenger bacteria that take advantage of the tumor environment instead of initiating $\mathrm{CRC}$, whereas the driver species that initiate CRC have been replaced by passenger bacteria due to the alteration of the intestinal niche.

Methods: Here, to reveal potential driver and passenger bacteria during CRC progression, we compare the gut mucosal microbiomes of 75 triplet-paired CRC samples collected from on-tumor site, adjacent-tumor site, and off-tumor site, and 26 healthy controls.

Results: Our analyses revealed potential driver bacteria in four genera and two families, and potential passenger bacteria in 14 genera or families. Bacillus, Bradyrhizobium, Methylobacterium, Streptomyces, Intrasporangiaceae and Sinobacteraceae were predicted to be potential driver bacteria. Moreover, 14 potential passenger bacteria were identified and divided into five groups. Group I passenger bacteria contain Fusobacterium, Campylobacter, Streptococcus, Schwartzia, and Parvimonas. Group II passenger bacteria contain Dethiosulfatibacter, Selenomonas, Peptostreptococus, Leptotrichia. Group III passenger bacteria contain Granulicatella. Group IV passenger bacteria contain Shewanella, Mogibacterium, and Eikenella. Group V passenger bacteria contain Anaerococus. Cooccurrence network analysis reveals a low correlation relationship between driver and passenger bacteria in CRC patients compared with healthy controls.

Discussion: These driver and passenger species may serve as bio-marker species for screening cohorts with high risk to initiate CRC or patients with CRC, respectively. Further functional studies will help understand the roles of driver and passenger bacteria in CRC initiation and development.

Keywords: colorectal cancer, driver-passenger model, microbiota

\section{Introduction}

Although it has been well accepted that genetic and epigenetic alterations are driving forces in carcinogenesis and progression of colorectal cancer (CRC), accumulated evidences suggest that specific microbial compositions in the intestine environment are frequently associated with CRC. For decades, development of high-throughput sequencing technologies facilitates delineating the catalogue of intestinal microbiota. More than 1,000 microbial species from the three domains of life have been identified in the human gastrointestinal tract, based on the analyses
Tianjin Union Medical Center, No. 190, Jieyuan Road, Hongqiao District, Tianjin City, People's Republic of China Email junliu_sci@I63.com

Xuehua Wan

Nankai University, No. 94, Weijin Road,

Nankai District, Tianjin City, People's

Republic of China

Email xuehua.wan@hotmail.com 
of their small subunit ribosomal RNA gene sequences. ${ }^{1,2}$ Currently, the most detailed report to date suggests that we are only approximately $47 \%$ human on average by cell count. ${ }^{3}$ Thus, such enormously diversified microbiota with enormous numbers comprise a complex ecosystem that contributes to the host metabolism in the gastrointestinal tract as well as host health and disease. It has been proposed that intestinal microbiota may play key roles in driving CRC. However, dominant bacterial taxa associated with $\mathrm{CRC}$ and their roles and potential mechanisms in CRC evolution remain to be completely understood.

Tjalsma et $\mathrm{al}^{4}$ proposed a bacterial driver-passenger model for $\mathrm{CRC}$, in which pathogenic bacteria initiating CRC development may be transiently colonized and replaced by other bacterial species with competitive growth advantage in the tumor niche. ${ }^{4}$ The former is defined as driver bacterium, and the latter is defined as passenger bacterium. ${ }^{4}$ Candidate driver bacteria show procarcinogenic features such as production of DNAdamaging compounds, interruption of tumor suppressor protein function, and induction of host inflammatory response. $^{5,6}$ These bacteria may colonize with low relative abundance but persistently interact with host cells to induce asymptomatic but chronic change in normal intestinal epithelium to initiate $\mathrm{CRC}^{7}$ Candidate passenger bacteria may show low relative abundances at the normal intestinal epithelium or the early stage of CRC, but competitively proliferate in the latter tumor niche. ${ }^{8}$ They take advantage of altered metabolism of tumor cells and outcompete the abundances of driver bacteria during development of CRC. These bacteria are hard to colonize on healthy colon tissue and cannot breach the intact colon wall. But they can easily invade a broken colon wall of adenoma or carcinoma. ${ }^{4}$ For example, Fusobacterium spp. appear to be the most common passenger bacteria in CRC. ${ }^{9}$ These passenger bacteria may continue contributing to the development of CRC. Survey of driver and passenger bacteria may serve as taxonomic bio-markers for determination of cohort with high risk to initiate $\mathrm{CRC}$ or patients with $\mathrm{CRC}$, respectively.

In this work, to identify potential driver and passenger bacteria in CRC evolution, we compare the gut mucosal microbiomes of 75 triplet-paired CRC samples collected from on-tumor site, adjacent-tumor site, and off-tumor site, and 26 healthy controls, which were obtained in Tianjin Union Medical Center. Because tumorigenesis is a chronic process in a localized position, off-tumor sites may represent the normal intestinal epithelium before the patients got CRC. Thus, highly abundant bacterial taxa associated with off-tumor sites may function as potential driver bacteria, which is presumed to have higher abundance in patients before they got CRC than healthy controls. On the other hand, highly abundant bacteria taxa associated with on-tumor sites may function as passenger bacteria. Using these criteria, we identify four genera and two families as potential driver bacteria, and 14 genera or families as potential passenger bacteria.

\section{Materials and Methods}

\section{Sample Collection}

We analyzed intestinal microbiome samples collected from 75 patients and 26 healthy controls in Tianjin Union Medical Center. The patient information about age and sex is listed in Table 1. Written informed consent was obtained from all participants prior to their inclusion in the study. All protocols and procedures of this study were approved by the Medical Ethics Board of Tianjin Union Medical Center. Three locations in the same removed tissue of a cancer patient were collected, including ontumor site $(\mathrm{T})$, adjacent-tumor site $(\mathrm{P})$ and off-tumor site $(\mathrm{N})$. Cotton swabs were used to dip into the intestinal surface of removed colorectal cancer tissues to collect the tumor and adjacent flora. All off-tumor sites were above the tumor to avoid contamination. Furthermore, intestinal microflora samples on intestinal mucosa of healthy people were also collected by colonoscopy as healthy samples $(\mathrm{H})$. Bacterial DNA of these samples was extracted using ZR Fungal/Bacterial DNA Kit according to the manufacturer's instructions.

\section{I6S rRNA Sequence Analysis}

The 16S ribosomal RNA amplicon libraries were constructed according to the Illumina manufacturer's manual. Briefly, the following primers were used to amplify the V3-V4 region of 16S rRNA gene: forward primer (PCR1_Forward), 5'TC GTCGGCAGCGTCAGATGTGTATAAGAGACAGCCTACGGGNGGCWGCAG and reverse primer (PCR1_Reverse),

Table I The Age and Gender Information of Patients in the Study

\begin{tabular}{|l|l|l|}
\hline & Patients (75) & Healthy Control (26) \\
\hline $\begin{array}{l}\text { Age (mean, range) } \\
\text { Male/female }\end{array}$ & $63.4(29-82)$ & $51.7(2 \mathrm{I}-7 \mathrm{I})$ \\
$48 / 27$ & $17 / 9$ \\
\hline
\end{tabular}




\section{5'GTCTCGTGGGCTCGGAGATGTGTATAAGAGACAG-}

GACTACHVGGGTATCTAATCC. Amplified DNA library was subsequently purified using AMPure XP beads and quantified using Quant-iT PicoGreen dsDNA assay kit (Invitrogen). The paired-end sequencing reads were generated on Illumina MiSeq platform. Quality control and filtering of raw sequences were carried out using FastQC (https://www. bioinformatics.babraham.ac.uk/projects/fastqc/). The filtered paired-end reads were assembled using PandaSeq. ${ }^{10}$ The assembled sequences were loaded on QIIME $^{11}$ for OTU picking, taxonomic assignment, and diversity analyses. Rarefaction curves were analyzed using alpha_rarefaction.py command from the QIIME pipeline (Supplementary Figure 1). The 16S rRNA sequencing reads have been submitted to the NCBI SRA database under accession number PRJNA606879. All the data are provided in this paper and supplementary Figure 1.

\section{Determination of Potential Driver and Passenger Bacteria}

Relative abundance of bacterial taxa was determined using arcsine square root. ${ }^{12}$ Candidate driver bacteria were determined when the relative abundance at an off-tumor site (N-site) was significantly higher than those at an on-tumor site (T-site). Candidate passenger bacteria were determined when its relative abundance at an on-tumor site (T-site) was significantly higher than those at an off-tumor site (N-site) and healthy controls $(\mathrm{H})$. The statistical significances of multiple samples comparisons were calculated using one-way ANOVA with Kruskal-Wallis test. ${ }^{*} P<0.05,{ }^{* *} P<0.01,{ }^{* * *} P<0.001, * * * * P<0.0001$.

\section{Correlation Network Analysis}

To calculate Spearman rank correlation values between bacterial taxa, taxa summary from QIIME output was loaded in $\mathrm{R}$ package Hmisc (Harrell, Vanderbilt University School of Medicine, Nashville, TN, USA). Correlation network analysis of the Spearman rank correlation values was carried out using Cytoscape 3.7.2. ${ }^{13}$

\section{Statistical Analysis}

Statistical analyses were performed using statistical analysis in social science program (SSPS). The statistical significances of multiple sample comparisons were calculated using one-way ANOVA with Kruskal-Wallis test. $* P<0.05,{ }^{*} * P<0.01, * * * P<0.001,{ }^{*} * * * P<0.0001$.

\section{Ethics Statement}

Research was conducted according to the principles expressed in the Declaration of Helsinki. The study was approved by the Ethics Committee of Tianjin Union Medical Center. Patients provided written informed consent for the collection of samples and subsequent analysis when required.

\section{Results}

\section{Determination of Potential Driver Bacteria in CRC}

To determine potential driver bacteria in CRC initiation, we surveyed relative abundances of bacterial taxa at genus level at an on-tumor site (T), an adjacent-tumor site $(\mathrm{P})$, an off-tumor site $(\mathrm{N})$, and in healthy controls (H) (Figure 1). We identified Bacillus, Bradyrhizobium, and Methylobacterium, with high levels of relative abundance (average $>1 \%$ ) as potential driver bacteria, whose relative abundance at off-tumor sites $(\mathrm{N})$ were significantly higher than those at on-tumor sites (T) and in healthy controls $(\mathrm{H})$ (Figure 2A-C). Moreover, relative abundances of these three genera at adjacent-tumor site (P) are significantly higher than those at on-tumor sites (T), respectively (Figure 2A-C). Particularly, the relative abundance of Bradyrhizobium at adjacent-tumor sites (P) was similar to that at off-tumor sites (N) and significantly higher than that in healthy controls $(\mathrm{H})$ (Figure 2B). These data indicate that these bacteria have a competitive growth advantage and outcompete other species in colonizing the intestinal niche of patients and may contribute to CRC initiation.

We also identified Streptomyces and two families, Intrasporangiaceae and Sinobacteraceae, with high levels of relative abundance, whose relative abundance at off-tumor sites $(\mathrm{N})$ and adjacent-tumor sites $(\mathrm{P})$ were both significantly higher than those at on-tumor sites (T) (Figure 2D-F). Their average relative abundance at off-tumor sites $(\mathrm{N})$ were higher than those from healthy controls $(\mathrm{H})$ but without statistical significance (Figure 2D-F). Moreover, the relative abundance of Streptomyces and Intrasporangiaceae in healthy controls $(\mathrm{H})$ were significantly higher than those at ontumor sites (T) (Figure 2D and F). We also considered these bacterial taxa as potential driver bacteria.

\section{Determination of Potential Group I Passenger Bacteria in CRC}

We next investigated potential passenger bacteria in CRC by filtering the relative abundance at on-tumor sites $(\mathrm{T})$ which 


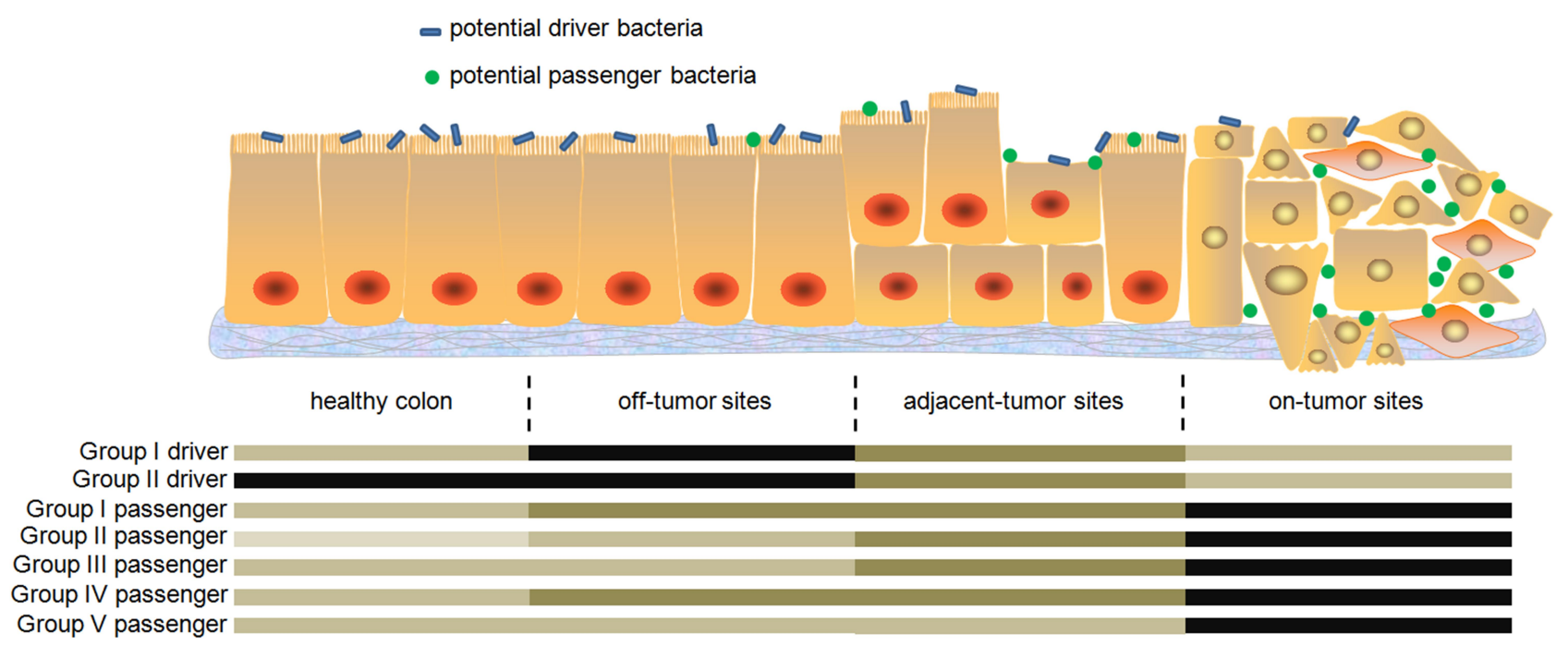

Figure I Schematic showing the off-tumor site, adjacent-tumor site, and on-tumor site in the intestinal tract of a CRC patient. Driver bacteria but not passenger bacteria can easily attach to intact colon wall at off-tumor sites. The latter are good colonizers at on-tumor sites and can invade distorted structure of carcinoma tissue. Relative abundances of each group are shown below the image, in which deep color indicates high abundance and light color indicates low abundance.

A

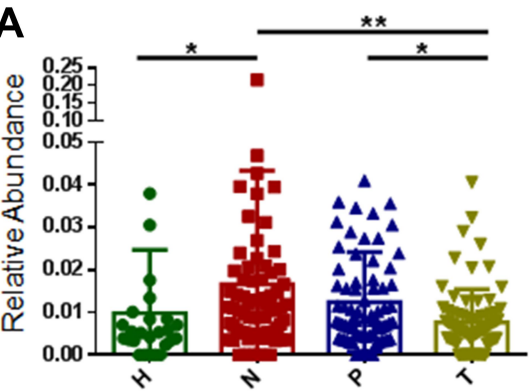

D

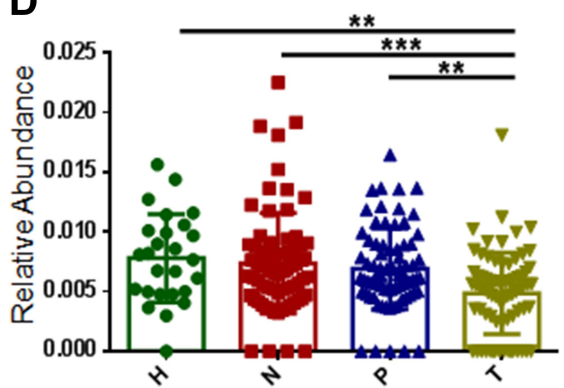

B

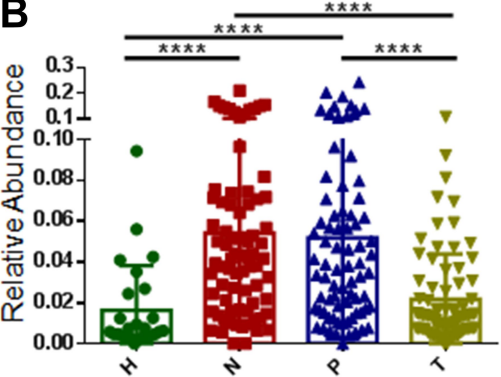

$\mathbf{E}$

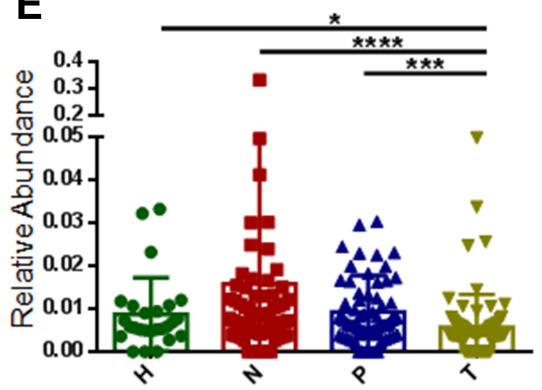

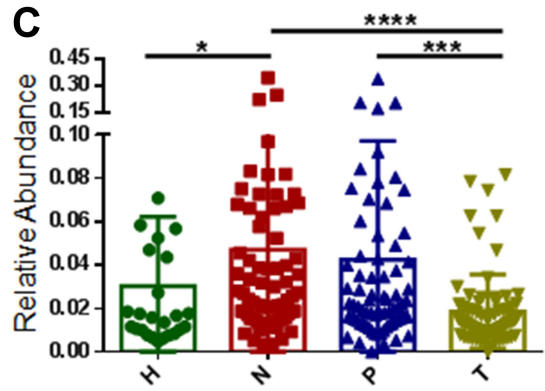

$\mathbf{F}$

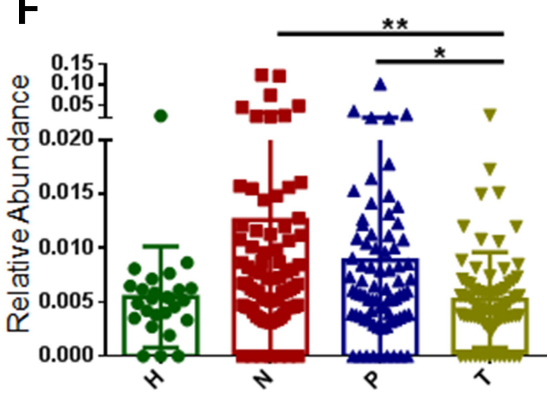

Figure 2 Identification of potential driver bacteria associated with CRC. Bacillus (A), Bradyrhizobium (B), Methylobacterium (C), Streptomyces (D), Intrasporangiaceae (E), and Sinobacteraceae $(\mathbf{F})$ as potential driver bacteria. $\mathrm{H}$ : healthy control, $\mathrm{N}$ : off-tumor site, P: adjacent-tumor site, T: on-tumor site. The statistical significances of multiple sample comparisons were calculated using one-way ANOVA with Kruskal-Wallis test. $* P<0.05, * * P<0.01, * * * P<0.001, * * * * P<0.000$ I.

were higher than those at off-tumor sites $(\mathrm{N})$. Based on the differential relative abundances of bacterial taxa at different sites, we divided the potential passenger bacteria into five groups. Group I passenger bacteria contained Fusobacterium, Campylobacter, Streptococcus, Schwartzia, and Parvimonas, whose relative abundances at on-tumor sites $(\mathrm{T})$ were higher than those at off-tumor sites $(\mathrm{N})$ and healthy controls $(\mathrm{H})$ (Figure $3 \mathrm{~A}-\mathrm{E}$ ). Relative abundances of
Campylobacter and Parvimonas at off-tumor sites (N) and adjacent-tumor sites $(\mathrm{P})$ were significantly higher than those from healthy controls $(\mathrm{H})$ (Figure $3 \mathrm{~B}$ and $\mathrm{E}$ ), suggesting that the whole intestinal niche of patients is different from that of healthy people and can be considered for screening passenger bacteria by comparing their abundance distributions at on- and off-tumor sites. Particularly, the relative abundances of Fusobacterium, Campylobacter, and Schwartzia at 
adjacent-tumor sites $(\mathrm{P})$ were significantly lower than those at on-tumor sites (T) (Figure 3A, B and D).

\section{Determination of Potential Group II, III, IV, and $\mathrm{V}$ Passenger Bacteria in CRC}

Groups II, III, IV and V passenger bacteria did not meet the criteria for group I, but their relative abundances at on-tumor sites $(\mathrm{T})$ were significantly higher than those at either offtumor sites $(\mathrm{N})$ or in healthy controls $(\mathrm{H})$ (Figure 4A-I). Group II passenger bacteria contained Dethiosulfatibacter, Selenomonas, Peptostreptococus, and Leptotrichia, whose relative abundances in healthy controls $(\mathrm{H})$ were significantly lower than those at off-tumor sites $(\mathrm{N})$, adjacenttumor sites $(\mathrm{P})$, and on-tumor sites $(\mathrm{T})$, suggesting that these bacteria may be inhibited by probiotics or other factors in heathy intestinal environments (Figure 4A-D). Group III passenger bacteria contain Granulicatella, whose relative abundance in healthy controls $(\mathrm{H})$ was significantly lower than that at adjacent-tumor sites $(\mathrm{P})$ and on-tumor sites $(\mathrm{T})$, suggesting that the integrity of the colon wall may be the main factor affecting the growth of these bacteria (Figure 4E). Group IV passenger bacteria contained Shewanella, Mogibacterium, and Eikenella, whose relative abundances in healthy controls $(\mathrm{H})$ were lower than those at on-tumor sites $(\mathrm{T})$ (Figure $4 \mathrm{~F}-\mathrm{H}$ ), indicating that these bacteria were enriched at on-tumor sites. Group V passenger bacteria contained Anaerococus, whose relative abundance at ontumor sites $(\mathrm{T})$ was significantly higher than that at offtumor sites (P) (Figure 4I), suggesting that these bacteria were prone to colonize on interrupted colon walls. Taken together, the niche of on-tumor sites is the most suitable environment for these groups of passenger bacteria to proliferate.

\section{Co-Occurrence Network of Potential Driver and Passenger Bacteria in CRC}

Bacteria in mixed-species populations co-exist to function in the progression of CRC. We next preformed correlation analysis of the potential driver and passenger bacteria identified above. Spearman rank correlation was carried out using relative abundances of bacterial taxa in healthy controls $(\mathrm{H})$, off-tumor sites $(\mathrm{N})$, adjacent-tumor sites $(\mathrm{P})$, and on-tumor sites $(\mathrm{T})$, respectively. All the correlation relationships were positive, while none of the negative correlation relationships was found between any potential driver and passenger bacteria. Interestingly, the potential driver bacteria defined above are highly correlated in all types of samples, respectively (Figure 5A-D). By contrast, merely a few potential passenger bacteria were correlated, especially in off-tumor sites $(\mathrm{N})$, adjacent-tumor sites $(\mathrm{P})$, and on-tumor sites (T) (Figure 5B-D). In healthy controls, certain potential driver and passenger bacteria were
A
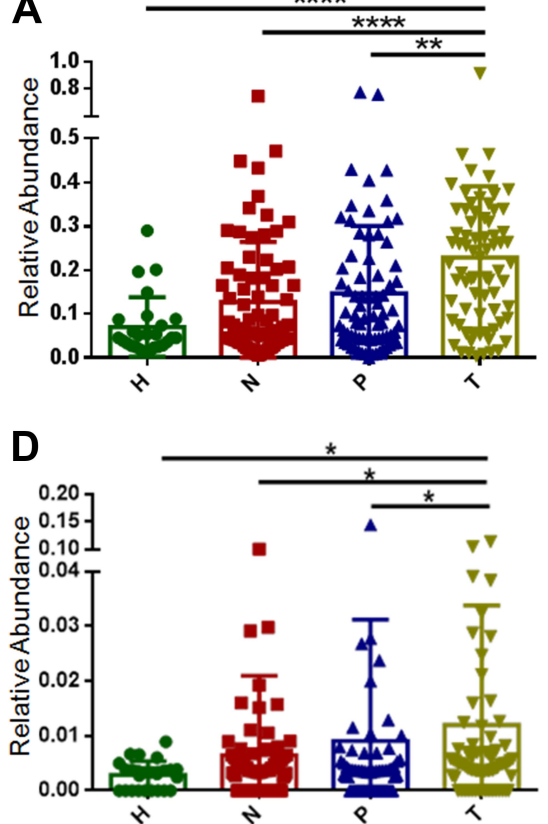
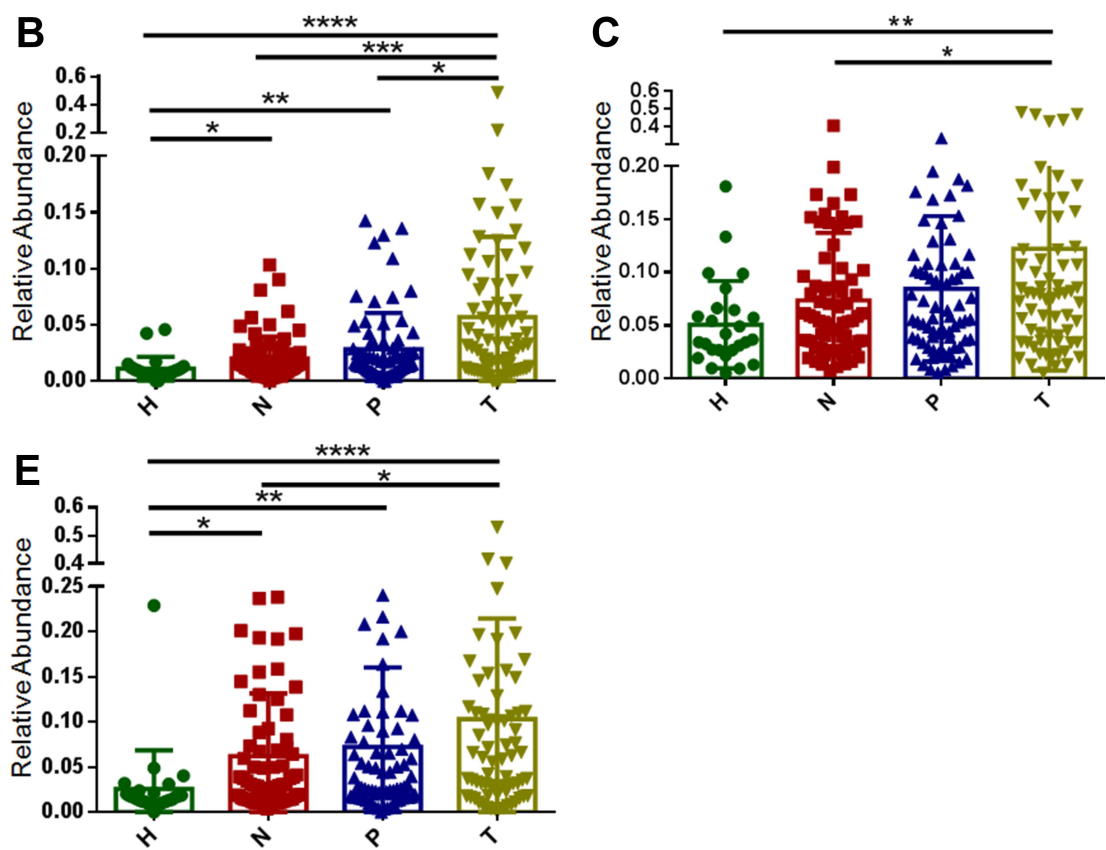

Figure 3 Identification of potential group I passenger bacteria associated with CRC. Fusobacterium (A), Campylobacter (B), Streptococcus (C), Schwartzia (D), and Parvimonas (E) as potential group I passenger bacteria. $\mathrm{H}$ : healthy control, $\mathrm{N}$ : off-tumor site, $\mathrm{P}$ : adjacent-tumor site, $\mathrm{T}$ : on-tumor site. The statistical significances of multiple sample comparisons were calculated using one-way ANOVA with Kruskal-Wallis test. $* P<0.05, * * P<0.01, * * * P<0.001, * * * * P<0.0001$. 

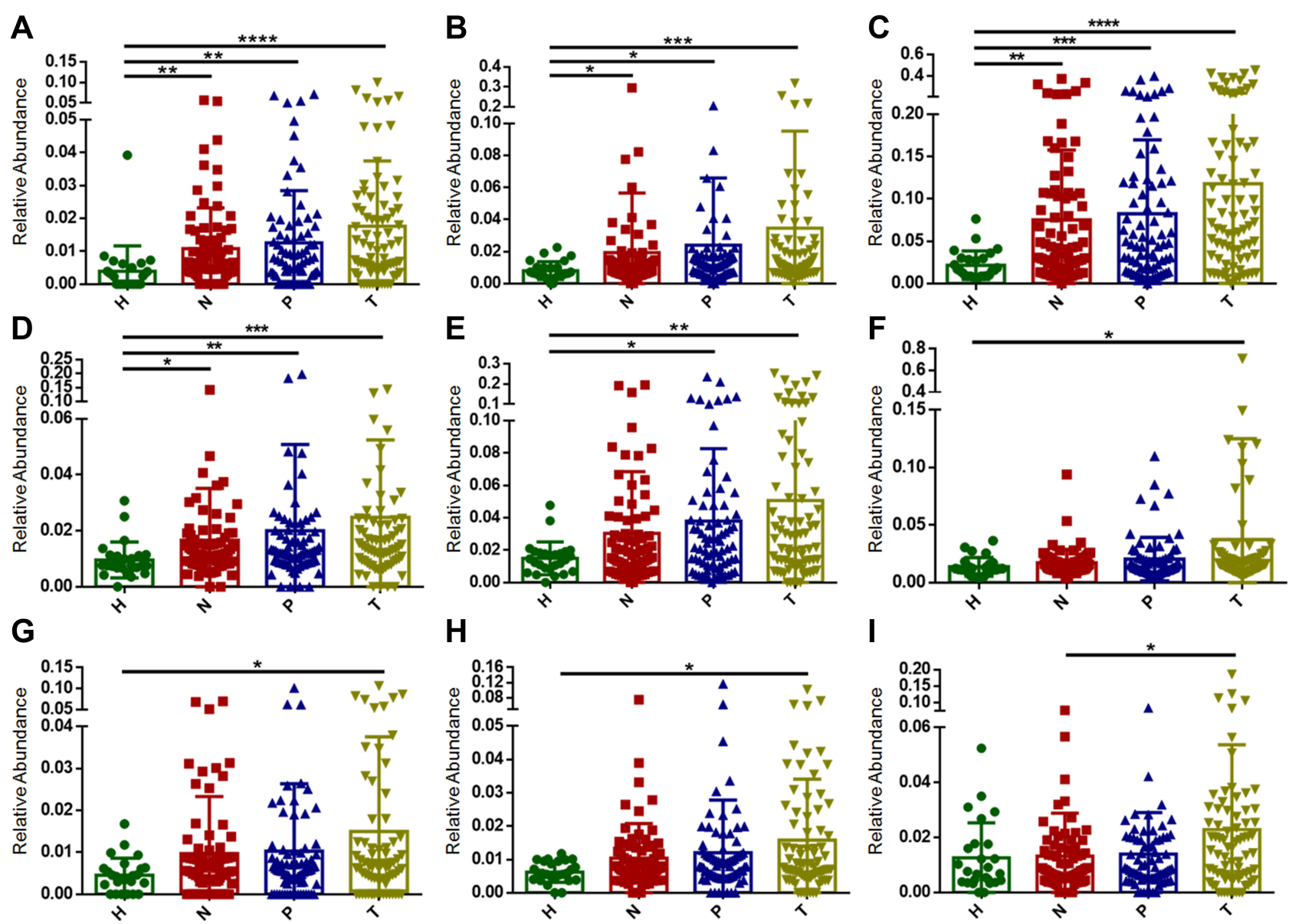

Figure 4 Identification of potential group II (A-D), III (E), IV (F-H), and V (I) passenger bacteria associated with CRC. Dethiosulfatibacter (A), Selenomonas (B), Peptostreptococus (C), and Leptotrichia (D) as potential group II passenger bacteria. Granulicatella (E) as a potential group III passenger bacteria. Shewanella (F), Mogibacterium (G), and Eikenella $(\mathbf{H})$ as potential group IV passenger bacteria. Anaerococus (I) as a potential group $V$ passenger bacteria. H: healthy control, $\mathrm{N}$ : off-tumor site, P: adjacent-tumor site, T: on-tumor site. The statistical significances of multiple sample comparisons were calculated using one-way ANOVA with Kruskal-Wallis test. $* P<0.05, * * P<0.01, * * * P<0.001, * * * * P<0.0001$.

positively correlated, suggesting that their abundances were balanced in healthy people. During initiation and development of $\mathrm{CRC}$, abundance levels of most driver bacteria and passenger bacteria were differently correlated.

\section{Discussion}

In this work, to reveal potential driver bacteria for CRC initiation and passenger bacteria for CRC development, we analyzed microbiomes of CRC patients and healthy controls in Tianjin Union Medical Center. We identified four genera including Bacillus, Bradyrhizobium, Methylobacterium, and Streptomyces, and two families including Intrasporangiaceae and Sinobacteraceae as potential driver bacteria. We identified 14 potential passenger bacteria and divided them into five groups. Group I passenger bacteria contained Fusobacterium, Campylobacter, Streptococcus, Schwartzia, and Parvimonas. Group II passenger bacteria contained Dethiosulfatibacter, Selenomonas, Peptostreptococus, Leptotrichia. Group III passenger bacteria contained Granulicatella. Group IV passenger bacteria contained Shewanella, Mogibacterium, and Eikenella. Group V passenger bacteria contained Anaerococus.

The potential driver bacteria of CRC defined in this study were found to be associated with other types of cancer as well. The analysis of $16 \mathrm{~S}$ rRNA showed a higher relative abundance of Bacillus spp. in breast cancer patients compared with healthy samples. ${ }^{14}$ The relative abundance of Bradyrhizobium japonicum was increased in lung cancer patients with early-stage tumors compared with those in patients with advanced-stage tumors (I \& II vs III \& IV). ${ }^{15}$ The analysis of V1-V2 region of $16 \mathrm{~S}$ rRNA showed that Methylobacteriaceae was abundant in malignant disease compared to benign disease in breast cancer patients. ${ }^{16}$ An intra-tumoral microbiome signature including Streptomyces and Bacillus clausii is highly predictive of long-term survivorship. ${ }^{17}$ Further physiological and functional studies 

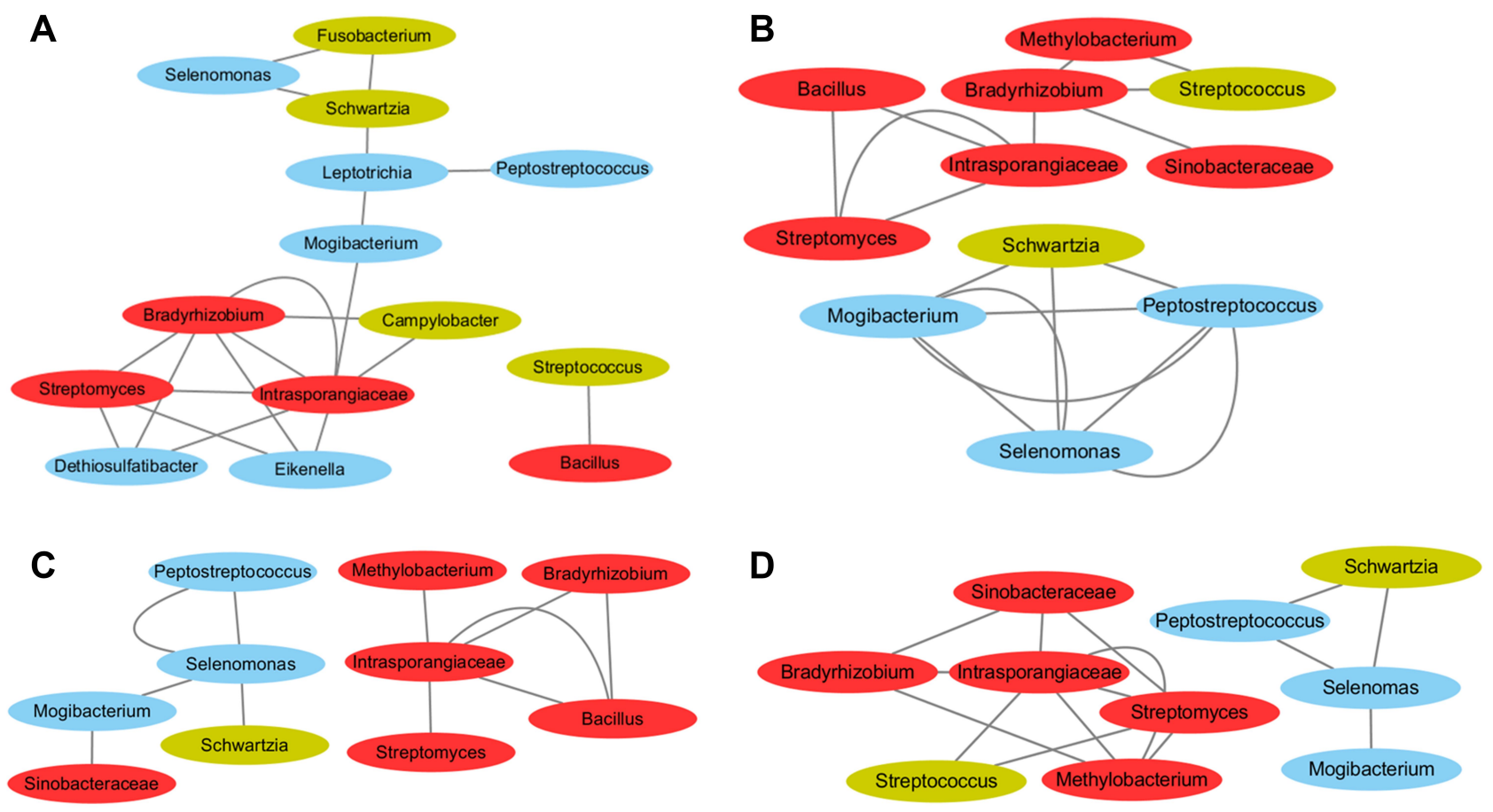

Figure 5 Correlation relationships among driver bacteria and passenger bacteria in healthy controls (A), off-tumor sites (B), adjacent-tumor sites (C), and on-tumor sites (D). Bacteria pairs with Spearman rank correlation above 0.50 were represented in the network. Edges represent correlation relationships between bacteria pairs. Red, potential driver bacteria. Green, potential group I passenger bacteria. Blue, potential group II, III, IV, and V passenger bacteria.

of representative pathogenic bacteria among these potential driver bacteria will help understand their roles in CRC initiation.

The majority of the potential passenger bacteria defined here were also found to be associated with CRC in previous reports. Fusobacterium, Peptostreptococcus, Parvimonas, Leptotrichia, Campylobacter, Streptococcus, Selenomas, Shewanella, Mogibacterium, and Eikenella were detected to be highly associated with CRC. ${ }^{18-25}$ Due to their high relative abundances in CRC samples, they have received more attention than driver bacteria and some of these CRCassociated bacteria were under investigation for their physiological roles in CRC evolution. For example, Fusobacterium spp., anaerobic Gram-negative bacteria, are highly associated with $\mathrm{CRC} .^{8,18,26}$ Particularly, Fusobacterium nucleatum has been identified to be an invasive and proinflammatory agent for acute and chronic oral and gastrointestinal infections. ${ }^{18,27-31}$ In addition, Fusobacterium spp. may function as a vector for host tissue infection by co-adherent bacteria. $^{18}$ Treatment of Fusobacterium with metronidazole (Fusobacteriumkilling antibiotic) reduced tumor-growth in vivo, ${ }^{21}$ suggesting that Fusobacterium contributes to CRC growth and metastasis. Campylobacter jejuni produces a genotoxin, cytolethal distending toxin, which has DNAse activity and causes DNA double-strand breaks. C. jejuni promotes CRC and induces changes in microbial composition. ${ }^{32}$ Fusobacterium spp. have been observed in significant cooccurrence with Leptotrichia and Campylobacter species, ${ }^{18}$ or with Bacteroides, Selenomonas, and Prevotella species. $^{21}$

Our co-occurrence network analysis of potential driver and passenger bacteria suggests bacterial compositions in different sample types show specific correlation patterns. Six driver bacteria taxa were highly correlated together in off-tumor sites. Sinobacteraceae was clustered with passenger bacteria in adjacent-tumor sites, while Bacillus was not correlated with either driver bacteria or passenger bacteria in on-tumor sites. In healthy samples, Bacillus, Bradyrhizobium, Streptomyces, and Intrasporangiaceae were correlated with passenger bacteria, while Methylobacterium and Sinobacteraceae were not correlated with any of them. Four passenger bacteria including Schwartzia in group I and Mogibacterium, Selenomonas, and Peptostreptococcus in groups II-V were highly correlated in off-tumor sites, adjacent-tumor sites, and on-tumor sites. Fusobacterium and Campylobacter were correlated with other driver and passenger bacteria in healthy samples, but none of them in off-tumor sites, adjacent-tumor sites, or on-tumor sites, suggesting that relative abundances of these 
potential driver and passenger bacteria were more balanced in healthy controls than CRC patients. Reduced bacterial composition in the intestine is the key feature for many intestinal and extra-intestinal disorders. ${ }^{33}$ It is suggested that a combination of alterations, rather than changed abundance of a particular strain in the intestinal microbiota promotes tumorigenesis. ${ }^{34}$ A high diversity with balanced driver and passenger bacteria might reflect a key feature of a healthy gut enabling a species-rich ecosystem to deal with environmental stresses that promote CRC. These mixed-species populations may interact, cooperate, or compete with each other in the intestinal ecosystem. These potential driver and passenger bacteria confirmed in this study are only based on bioinformatics analysis, so how microbial diversity protects against tumorigenesis and how driver bacteria initiate $\mathrm{CRC}$ and are replaced by passenger bacteria in the later stage remain to be further investigated. Furthermore, the precise role of these bacteria on the intestinal tract need to be revealed by further experiments in vitro and in vivo, especially for those predicted as passenger bacteria. Passenger bacteria have high relative abundances in tumor niches, but some of them may act as probiotics, which has the function of establishment of balanced intestinal microflora, synthesis of vitamins and inactivation of cancerogenic compounds. ${ }^{35,36}$

Taken together, this study reports potential driver and passenger bacteria identified in human CRC. Further retrospective study will help identify potential driver and passenger bacteria as well. In addition, it is important to investigate whether driver bacteria identified in off-tumor sites of CRC patients are also present in the fecal and colonoscopy samples of CRC patients. Only a few driver bacteria have been identified based on experimental data in vitro and in vivo, ${ }^{5}$ and many potential driver bacteria need to be found and identified. Furthermore, abundances of driver and passenger bacteria may serve as a reference biomarker in clinical diagnosis of CRC initiation risk and development. These potential driver and passenger bacteria found in our study provide a preliminary scope to carry out further investigations into the mechanisms involved.

\section{Funding}

This research was funded by the Natural Science Foundation of China Grant number 81972826, and the Fundamental Research Funds for the Central Universities, Nankai University Grant number 63191440, and Key R\&D Projects in the Tianjin Science and Technology Pillar Program Grant number 19YFZCSY00420, and National key R\&D Program of China Grant number 2017YFC1700604, and National key R\&D Program of China Grant number 2017YFC1700606, and Tianjin 131 Innovative Talent Training Project in 2018, and the Research Foundation of Tianjin Union Medical Center number 2018YJ023.

\section{Disclosure}

The authors report no conflicts of interest in this work.

\section{References}

1. Qin J, Li R, Raes J, et al. Europe PMC Funders Group Author Manuscripts. A human gut microbial gene catalog established by metagenomic sequencing. Nature. 2010;464:59-65. doi:10.1038/ nature 08821

2. Rajilić-Stojanović M, de Vos WM. The first 1000 cultured species of the human gastrointestinal microbiota. FEMS Microbiol Rev. 2014;38:996-1047. doi:10.1111/1574-6976.12075

3. Knight R, Callewaert C, Marotz C, et al. The microbiome and human biology. Annu Rev Genomics Hum Genet. 2017;18:65-86. doi:10.1146/annurev-genom-083115-022438

4. Tjalsma H, Boleij A, Marchesi JR, Dutilh BE. A bacterial driver-passenger model for colorectal cancer: beyond the usual suspects. Nat Rev Micro. 2012;10:575-582. doi:10.1038/nrmicro2819

5. Rhee KJ, Wu SG, Wu XQ, et al. Induction of persistent colitis by a human commensal, enterotoxigenic bacteroides fragilis, in wild-type C57BL/6 mice. Infect Immun. 2009;77:1708-1718.

6. Kapral M, Węglarz L, Parfiniewicz B, et al. Quantitative evaluation of

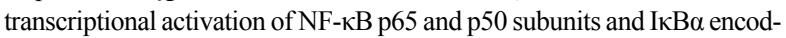
ing genes in colon cancer cells by desulfovibrio desulfuricans endotoxin. Folia Microbiol. 2010;55:657-661. doi:10.1007/s12223-010-0106-6

7. Gao Z, Guo B, Gao R, et al. Microbiota disbiosis is associated with colorectal cancer. Front Microbiol. 2015;6:20. doi:10.3389/ fmicb.2015.00020

8. Kostic AD, Gevers D, Pedamallu CS, et al. Genomic analysis identifies association of Fusobacterium with colorectal carcinoma. Genome Res. 2012;22:292-298. doi:10.1101/gr.126573.111

9. Amitay EL, Werner S, Vital M, et al. Fusobacterium and colorectal cancer: causal factor or passenger? Results from a large colorectal cancer screening study. Carcinogenesis. 2017;38:781-788. doi:10.1093/carcin/bgx053

10. Masella AP, Bartram AK, Truszkowski JM, Brown DG, Neufeld JD. PANDAseq: paired-end assembler for illumina sequences. $B M C$ Bioinformatics. 2012;13:31. doi:10.1186/1471-2105-13-31

11. Caporaso J, Kuczynski J, Stombaugh J, et al. QIIME allows analysis of high-throughput community sequencing data. Nat Methods. 2010;7:335-336. doi:10.1038/nmeth.f.303

12. Nakatsu G, Li X, Zhou H, et al. Gut mucosal microbiome across stages of colorectal carcinogenesis. Nat Commun. 2015;6:8727. doi:10.1038/ncomms 9727

13. Shannon P, Markiel A, Ozier O, et al. Cytoscape: a software environment for integrated models of biomolecular interaction networks. Genome Res. 2003;13:2498-2504. doi:10.1101/gr.1239303

14. Eslami SZ, Majidzadeh AK, Halvaei S, et al. Microbiome and breast cancer: new role for an ancient population. Front Oncol. 2020;10:1-15. doi:10.3389/fonc.2020.00120

15. Jin J, Gan Y, Liu H, et al. Diminishing microbiome richness and distinction in the lower respiratory tract of lung cancer patients: a multiple comparative study design with independent validation. Lung Cancer. 2019;136:129-135. 
16. Meng S, Chen B, Yang J, et al. Study of microbiomes in aseptically collected samples of human breast tissue using needle biopsy and the potential role of in situ tissue microbiomes for promoting malignancy. Front Oncol. 2018;8:318. doi:10.3389/fonc.2018.00318

17. Riquelme E, Zhang Y, Zhang L, et al. Tumor microbiome diversity and composition influence pancreatic cancer outcomes. Cell. 2019;178:795-806. doi:10.1016/j.cell.2019.07.008

18. Warren RL, Freeman DJ, Pleasance S, et al. Co-occurrence of anaerobic bacteria in colorectal carcinomas. Microbiome. 2013;1:1-12. doi:10.1186/2049-2618-1-16

19. Rajagopala SV, Vashee S, Oldfield LM, et al. The human microbiome and cancer. Cancer Prev Res. 2017;10:226-234. doi:10.1158/19406207.CAPR-16-0249

20. Bultman SJ. Emerging roles of the microbiome in cancer. Carcinogenesis. 2014;35:249-255. doi:10.1093/carcin/bgt392

21. Bullman S, Pedamallu CS, Sicinska E, et al. Analysis of Fusobacterium persistence and antibiotic response in colorectal cancer. Science. 2017;358:1443-1448. doi:10.1126/science.aal5240

22. Flemer B, Lynch DB, Brown JMR, et al. Tumour-associated and non-tumour-associated microbiota in colorectal cancer. Gut. 2017;66:633-643. doi:10.1136/gutjnl-2015-309595

23. Chen W, Liu F, Ling Z, et al. Human intestinal lumen and mucosa-associated microbiota in patients with colorectal cancer. PLoS One. 2012;7:e39743. doi:10.1371/journal.pone.0039743

24. Hale VL, Chen J, Johnson S, et al. Shifts in the fecal microbiota associated with adenomatous polyps. Cancer Epidemiol Biomarkers Prev. 2017;26:85-94.

25. Allali I, Delgado S, Marron PI, et al. Gut microbiome compositional and functional differences between tumor and non-tumor adjacent tissues from cohorts from the US and Spain. Gut Microbes. 2015;6:161-172. doi:10.1080/19490976.2015.1039223

26. Castellarin M, Warren RL, Freeman JD, et al. Fusobacterium nucleatum infection is prevalent in human colorectal carcinoma. Genome Res. 2012;22:299-306. doi:10.1101/gr.126516.111
27. Han YW, Shi W, Huang GT, et al. Interactions between periodontal bacteria and human oral epithelial cells: Fusobacterium nucleatum adheres to and invades epithelial cells. Infect Immun. 2000;68:3140-3146. doi:10.1128/IAI.68.6.3140-3146.2000

28. Krisanaprakornkit S, Kimball JR, Weinberg A, et al. Inducible expression of human beta-defensin 2 by Fusobacterium nucleatum in oral epithelial cells: multiple signaling pathways and role of commensal bacteria in innate immunity and the epithelial barrier. Infect Immun. 2000;68:2907-2915. doi:10.1128/IAI.68.5.2907-2915.2000

29. Peyret-Lacombe A, Brunel G, Watts M, et al. TLR2 sensing of F. nucleatum and $\mathrm{S}$. sanguinis distinctly triggered gingival innate response. Cytokine. 2009;46:201-210. doi:10.1016/j.cyto.2009.01.006

30. Signat B, Roques C, Poulet P, et al. Fusobacterium nucleatum in periodontal health and disease. Curr Issues Mol Biol. 2011;13:25-36.

31. Swidsinski A, Dörffel Y, Loening-Baucke V, et al. Acute appendicitis is characterised by local invasion with Fusobacterium nucleatum/ necrophorum. Gut. 2011;60:34-40. doi:10.1136/gut.2009.191320

32. He Z, Gharaibeh RZ, Newsome RC, et al. Campylobacter jejuni promotes colorectal tumorigenesis through the action of cytolethal distending toxin. Gut. 2019;68:289-300. doi:10.1136/gutjnl-2018317200

33. Tilg H, Kaser A. Gut microbiome, obesity, and metabolic dysfunction. J Clin Invest. 2011;121:2126-2132. doi:10.1172/ JCI58109

34. Tilg H, Adolph TE, Gerner RR, Moschen AR. The intestinal microbiota in colorectal cancer. Cancer Cell. 2018;33:954-964. doi:10.1016/j.ccell.2018.03.004

35. Uccello M, Malaguarnera G, Basile F, et al. Potential role of probiotics on colorectal cancer prevention. BMC Surg. 2012;12(Suppl 1): S35. doi:10.1186/1471-2482-12-S1-S35

36. MalaGuarnera G, leGGio F, Vacante $M$, et al. Probiotics in the gastrointestinal diseases of the elderly. $J$ Nutr Health Aging. 2012;16:402-410. doi:10.1007/s12603-011-0357-1
Cancer Management and Research

\section{Publish your work in this journal}

Cancer Management and Research is an international, peer-reviewed open access journal focusing on cancer research and the optimal use of preventative and integrated treatment interventions to achieve improved outcomes, enhanced survival and quality of life for the cancer patient.

\section{Dovepress}

The manuscript management system is completely online and includes a very quick and fair peer-review system, which is all easy to use. Visit http://www.dovepress.com/testimonials.php to read real quotes from published authors. 